\title{
Popular Kohn-Sham density functionals strongly overestimate many-body interactions in van der Waals systems
}

\author{
Alexandre Tkatchenko ${ }^{1, *}$ and O. Anatole von Lilienfeld ${ }^{2, \dagger}$ \\ ${ }^{1}$ Fritz-Haber-Institut der Max-Planck-Gesellschaft, Faradayweg 4-6, D-14195 Berlin-Dahlem, Germany \\ ${ }^{2}$ Multiscale Dynamic Materials Modeling Department, Sandia National Laboratories, Albuquerque, New Mexico 87185-1322, USA
}

(Received 23 May 2008; published 24 July 2008)

\begin{abstract}
We find spuriously large repulsive many-body contributions to binding energies of rare gas systems for the first three rungs of "Jacob's Ladder" within Kohn-Sham density functional theory. While the description of van der Waals dimers is consistently improved by the pairwise London $C_{6} / R^{6}$ correction, inclusion of a corresponding three-body Axilrod-Teller $C_{9} / R^{9}$ term only increases the repulsive error. Our conclusions based on extensive solid state and molecular electronic structure calculations are particularly relevant for condensed phase van der Waals systems.
\end{abstract}

DOI: 10.1103/PhysRevB.78.045116

PACS number(s): 71.15.Mb, 71.15.Nc, 31.50.Bc, 61.50.Lt

\section{INTRODUCTION}

Among the various ways to compute and employ for atomistic simulation interatomic forces from first principles, Kohn-Sham density functional theory ${ }^{1,2}$ (DFT) is one of the most popular tools. Its capability to quantitatively estimate potential energy hypersurfaces of gases, liquids, or bulk within the local density approximation ${ }^{3}$ (LDA), generalized gradient approximation ${ }^{4,5}$ (GGA), "meta"-generalized gradient approximation ${ }^{6}$ (meta-GGA), or hybrid approximation ${ }^{7}$ is typically outstanding. ${ }^{89}$ The accuracy of such DFT-based models of the electronic structure, however, depends not only greatly upon the deployed approximation to the exchange-correlation potential $\left(v_{x c}\right)$ but also upon the system which is being studied. ${ }^{8}$ The difficulties, for instance, in obtaining a reasonable DFT-based description of van der Waals interactions, the ubiquitous interatomic forces dominant in the case of rare gases, have been recognized for more than a decade. ${ }^{10,11}$ There are various approaches to tackle this problem within DFT, such as extending the exchange-correlation potential by a nonlocal van der Waals term, ${ }^{12,13}$ leveraging the optimized potential method within Kohn-Sham perturbation theory, ${ }^{14,15}$ empirically calibrating dispersion corrected atom centered potentials, ${ }^{16-20}$ or directly fitting exchangecorrelation potentials to data sets of weakly bonded compounds..$^{21}$

One of the simplest yet most effective remedies for this error, however, consists of an atomic force-field-like correction, namely to superimpose a dissociative pairwise interatomic potential decaying as $C_{6} / R^{6}$, due to the seminal work of London, ${ }^{22,23}$ on top of the atomic forces obtained from DFT via the Hellmann-Feynman theorem. This approach yields good results if damped appropriately. ${ }^{24,25}$ A wide variety of such implementations exist, typically being designed, tested, and employed for gas phase systems. ${ }^{26-34}$ For large cluster or bulk properties, however, interatomic many-body effects are typically non-negligible. ${ }^{35,36}$ For example, Rościszewski et al. ${ }^{37}$ predicted for rare gas crystals repulsive van der Waals type many-body contributions (MBCs) to the cohesive energy of $\approx 3,7,8$, and $9 \%$ for $\mathrm{Ne}, \mathrm{Ar}, \mathrm{Kr}$, and $\mathrm{Xe}$, respectively. It remains therefore questionable, as also found by Ortmann et al. ${ }^{31}$ if the proclaimed gain in accuracy due to a $C_{6}$ correction is sufficiently transferable for a "black- box" study of noncovalent bulk systems or surface adsorption, such as in Refs. 38-41, and if further many-body terms such as a three-body Axilrod-Teller expression ${ }^{42}$ decaying as $C_{9} / R^{9}$ might be required for DFT calculations of van der Waals crystals.

In this study, we address this question by elucidating the many-body effects on interatomic forces derived from DFT calculations of the gas and bulk phase of rare gas atoms. Rare gas atoms, from an interatomic potential point of view, represent a very fundamental yet realistic system in that they produce the simplest contribution to binding, namely the nondirectional van der Waals forces. We find an unexpectedly large and systematic overestimation of the MBC to the interatomic potential-when compared to experiment or quantum chemistry calculations at the level of coupled cluster with single, double, and perturbative triple excitations $[\operatorname{CCSD}(\mathrm{T})]$. Obviously this error cannot be mitigated by the pairwise $C_{6}$ correction. Furthermore, contrary to the well performing attractive $C_{6}$ correction for underestimated diatomic interaction energies, this many-body error would be worsened by a corresponding repulsive $C_{9}$ three-body Axilrod-Teller correction. ${ }^{42}$

We have observed this effect for the trimer and bulk across the first three rungs of "Jacob's ladder" of DFT functionals: LDA, GGA [Perdew-Burke-Ernzerhof $(\mathrm{PBE}),{ }^{4}$ revised Perdew-Burke-Ernzerhof (revPBE) (Ref. 5)], metaGGA [Tao-Perdew-Staroverov-Scuseria (TPSS) (Ref. 6)], and hybrid GGA [PBE0 (Ref. 7)] using various software implementations of DFT; details are given in the methodology section. We investigated Ar with most of the calculations because of its abundant experimental and theoretical documentation. In order to extend our conclusions to all rare gases, selected calculations have also been performed for $\mathrm{Ne}$ and $\mathrm{Kr}$, yielding similar results.

\section{METHODOLOGY}

\section{A. Definitions}

The two- and three-body interatomic energies have been defined for this study as, $E_{(2)}=E_{\mathrm{dim}}-2 E_{\mathrm{mon}}$, and $E_{(3)}=E_{\text {trim }}$ $-3 E_{(2)}-3 E_{\text {mon }}$, respectively, where trim, dim, and mon stand for the equilateral triangular trimer, dimer, and monomer. 
Other three-body interactions than equilateral triangular have not been considered as they are expected to be significantly smaller. In a complete analogy to the energy terms, we have defined the three-body electron density distribution as, $n_{(3)}(\mathbf{r})=n_{\text {trim }}(\mathbf{r})-3 n_{(2)}(\mathbf{r})-3 n_{\text {mon }}(\mathbf{r})$, with $n_{(2)}(\mathbf{r})$ being the two-body electron density, $n_{\text {dim }}(\mathbf{r})-2 n_{\text {mon }}(\mathbf{r})$.

We have estimated the MBC to the cohesive energy of the Ar crystal by subtracting the corresponding cohesive energies of an Ar crystal made up of two-body potentials as obtained from the Ar dimer, $E_{\mathrm{MBC}}=E_{\mathrm{coh}}-E_{\mathrm{coh}}^{(2)}$. Clearly, the MBC contains not only the equilateral triangular three-body term but also all other MBCs corresponding to other threebody geometries or higher body terms.

\section{B. Computational details}

Various electronic structure codes, approximations, and set-ups have been used for this study. DFT calculations for Ar have been carried out using the pseudopotential-based plane-wave basis set electronic structure program CPMD $3.11,{ }^{43}$ Goedecker's pseudopotentials from Refs. 44 and 45, a plane-wave kinetic energy cutoff of $200 \mathrm{Ry}$, and large unit cells of $17 \times 12 \times 12 \AA^{3}$ for the dimer and $17 \times 15 \times 12 \AA^{3}$ for the trimer. PBE pseudopotentials have been employed for revPBE, PBE0, and TPSS functionals. We have converged our results with respect to kinetic energy cutoff, unit cell size, and total energy convergence. For $\mathrm{Ne}$ and $\mathrm{Kr}$ atoms, DFT calculations were done with NWChem, ${ }^{46} \operatorname{CCSD}(\mathrm{T})$ with Gaussian03 (both using BSSE-corrected energies with the aug-cc-pV5Z basis set ${ }^{47,48}$ ). Typical deviations of our results from available literature amount to less than $0.2 \mathrm{meV}$, which is negligible for our conclusions. For the Ar dimer and trimer, we have verified our DFT results independently by comparison with Gaussian03 (Ref. 49) using aug-cc-pVQZ basis sets ${ }^{48}$ for LDA, PBE, and PBE0 functionals. Typical deviations between plane-waves and Gaussian basis set calculations were below $1 \mathrm{meV}$.

For all the cubic bulk (fcc) calculations, a $6 \times 6 \times 6$ Monkhorst-Pack grid has been employed for sampling of the irreducible Brillouin zone, the exception being the TPSS calculations, which were performed at the $\Gamma$ point only with 108 atoms per unit cell, using CPMD 3.11. ${ }^{43}$ The PBE0 calculations have been done with VASP $5.1,{ }^{50}$ using a $3 \times 3 \times 3$ Monkhorst-Pack grid for a four-atom unit cell.

The London dispersion $C_{6} / R^{6}$ correction employed here builds up on previous work by Grimme ${ }^{51}$ and by Jurecka et $a l .{ }^{52}$ We have reoptimized the parameters of the correction scheme $\left(s_{6}, s_{R}\right)$ (see Ref. 53 for more details) for PBE, revPBE, PBE0, and TPSS functionals in CPMD $\left[\left(s_{6}, s_{R}\right)\right.$ $=(1.00,1.00),(1.26,0.84),(1.00,1.04),(1.00,0.93)$, respectively] on high-quality [second-order Møller-Plesset (MP2) extrapolated to complete basis set size plus a $\operatorname{CCSD}(\mathrm{T})$ correlation energy correction] binding energy database from Ref. 54, which does not explicitly include rare gas systems. Although $s_{6}$ being different from unity is unphysical at long distances, since the $C_{6} / R^{6}$ term must converge to the correct limit, in practice it helps to approximately model the lack of higher-order terms in the van der Waals energy expansion for the equilibrium distance (for further details see Ref. 53). The

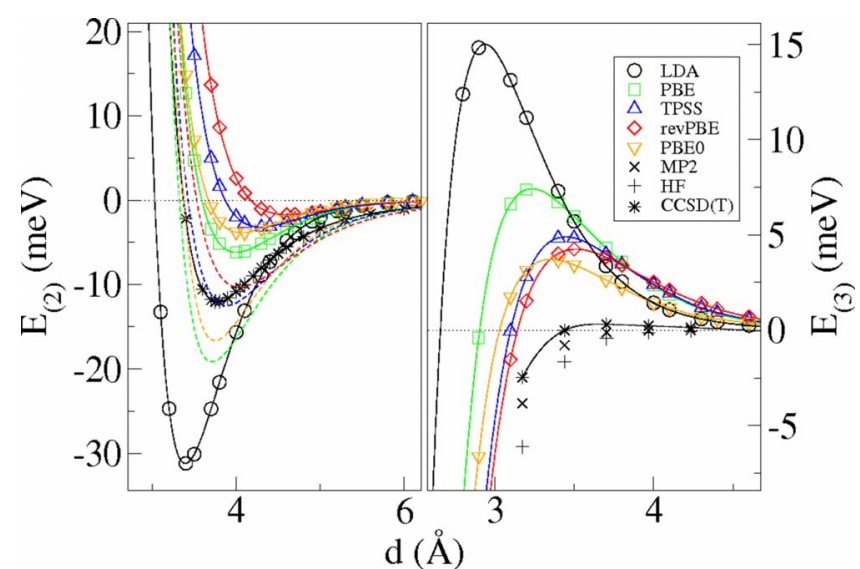

FIG. 1. (Color online) Left: two-body energy as function of interatomic distance for $\mathrm{Ar}_{2}$ with various functionals and $\mathrm{CCSD}(\mathrm{T})$ (Ref. 55). Dashed lines correspond to the $C_{6}$ corrected functionals. Right: three-body energies as a function of interatomic distance for the equilateral triangular $\mathrm{Ar}_{3} . \mathrm{CCSD}(\mathrm{T}), \mathrm{MP} 2$, and $\mathrm{HF}$ have been taken from Ref. 56.

$C_{6}$ coefficient $\left(C_{6}=75\right.$ a.u. $)$ for Ar has been fitted to highquality $\operatorname{CCSD}(\mathrm{T})$ data of Ref. 55 and is in good agreement with other estimates in the literature. ${ }^{51}$

\section{RESULTS AND DISCUSSION}

\section{A. $\mathrm{Ar}_{2}$ and $\mathrm{Ar}_{3}$}

The two- and three-body interatomic energies of $\mathrm{Ar}_{2}$ and $\mathrm{Ar}_{3}$ are displayed for various functionals as a function of distance in Fig. 1. Not surprisingly, the $C_{6}$ correction greatly improves the dimer results for all functionals, in particular for the meta-generalized gradient functional TPSS - as already foreseen in Ref. 57. The $\operatorname{CCSD}(\mathrm{T})$ three-body energy contribution is repulsive in the long range, has a small maximum of $\approx 0.3 \mathrm{meV}$ at $3.70 \AA$, crosses zero at $3.44 \AA$, and turns strongly attractive for the short interatomic range. While all the DFT functionals yield a qualitatively correct (repulsive) triangular three-body potential, ${ }^{42}$ its magnitude is drastically overestimated, and zero-point, maximum, and inflection-point are at far too short distances when compared to the CCSD(T) results. Climbing Jacob's ladder from LDA to GGA to meta-GGA does result into a continuous decrease in the error in the three-body potential, the hybrid PBE0, or the revPBE GGA, yielding similar results as the meta-GGA. Note that while the absolute magnitude of $E_{(3)}$ at its maximum is not large for $\mathrm{Ar}_{3}$ (at least $4 \mathrm{meV}$ ), we will show below that this error translates to $\geq 40 \mathrm{meV}$ for the cohesive energy of the bulk. When adding the artificially repulsive three-body potential to the over-attractive two-body potential, it becomes evident that it is only due to a fortuitous cancellation of errors when LDA describes total binding properties significantly better for $\mathrm{Ar}_{3}$ than for $\mathrm{Ar}_{2}$. Interestingly, while the Hartree-Fock (HF) and MP2 three-body energies both do not have a repulsive tail, their quantitative agreement with $\operatorname{CCSD}(\mathrm{T})$ is better than for all DFT results.

Corresponding equilibrium energy results, with and without the $C_{6}$ correction, feature in Table I. When applying the 
TABLE I. Ar equilibrium results for $C_{6}$ corrected and uncorrected functionals on "Jacob's ladder." Interatomic distances $d$ in dimer ( $\mathrm{dim}$ ) and fcc crystal (bulk) in $\AA$, corresponding dimer interaction energy $\left(E_{(2)}\right)$, triangular three-body nonadditive energy contribution $\left(E_{(3)}\right)$, two-body cohesive energy $\left(E_{\mathrm{coh}}^{(2)}\right)$, bulk many-body contribution $\left(E_{\mathrm{MBC}}\right)$, and cohesive energy $\left(E_{\mathrm{coh}}=E_{\mathrm{coh}}^{(2)}+E_{\mathrm{MBC}}\right)$ in $\mathrm{meV}$.

\begin{tabular}{lccccccc}
\hline \hline & $d_{\text {dim }}$ & $d_{\text {bulk }}$ & $E_{(2)}$ & $E_{(3)}$ & $E_{\text {coh }}^{(2)}$ & $E_{\mathrm{MBC}}$ & $E_{\text {coh }}$ \\
\hline LDA & 3.39 & 3.50 & -31.2 & 7.5 & -189.7 & 49.9 & -139.8 \\
PBE & 4.00 & 4.18 & -6.2 & 2.3 & -35.3 & 11.3 & -24.0 \\
revPBE & 4.61 & 4.85 & -1.7 & 0.7 & -9.3 & 2.6 & -6.7 \\
TPSS & 4.29 & 4.49 & -3.2 & 1.2 & -21.7 & 9.8 & -11.9 \\
PBE0 & 4.06 & 4.24 & -3.9 & 1.3 & -21.8 & 1.8 & -20.0 \\
PBE $+C_{6}$ & 3.71 & 3.72 & -19.1 & 4.2 & -146.4 & 31.7 & -114.7 \\
revPBE+ $+C_{6}$ & 4.03 & 3.99 & -10.3 & 2.4 & -86.3 & 18.4 & -67.9 \\
TPSS $+C_{6}$ & 3.89 & 3.91 & -12.5 & 3.0 & -97.2 & 22.9 & -74.3 \\
PBE0 $+C_{6}$ & 3.75 & 3.75 & -16.6 & 2.4 & -123.2 & 13.6 & -109.6 \\
Ref. & $3.76^{\mathrm{a}, \mathrm{b}}$ & $3.78^{\mathrm{c}}$ & $-12.3^{\mathrm{b}}$ & $0.3^{\mathrm{d}}$ & -94.3 & $6.3^{\mathrm{e}, \mathrm{f}}$ & $-88.0^{\mathrm{d}}$ \\
\hline \hline
\end{tabular}

${ }^{\mathrm{a}}$ Experimental, Reference 58

${ }^{\mathrm{b}}$ Experimental, Reference 59

${ }^{\mathrm{c}}$ Experimental, Theoretical, References 37 and 60

${ }^{\mathrm{d}}$ Theoretical, Reference 56

${ }^{e}$ Experimental (Reference 61) after removal of ZPE (Reference 37)

${ }^{f}$ Theoretical, Reference 55

attractive $C_{6}$ correction the error in the three-body contribution is aggravated by a factor of $\approx 2$ solely due to the shortening of interatomic distances-the reason being that $E_{(3)}$ has a negative and quite significant slope at the equilibrium distance, its maximum being at distances much shorter than the true equilibrium distance (see Fig. 1). For further insight, Fig. 2 features isosurfaces of the CCSD and LDA density difference, and of the LDA three-body electron density, $n_{(3)}(\mathbf{r})$. In the center of the system a major depletion of electron density occurs for both plots. The three-body density exhibits significant changes at each atomic site, which are reminiscent of angular momentum dependent functions. This indicates that not only the two but also the three-body DFT error is due to an overly delocalized electron density.

\section{B. Ar-bulk}

For the bulk we find that the MBC error is much larger in magnitude while similar trends as in the three-body case persist. This is consistent with the observation that the triangular three-body term is the dominating $\mathrm{MBC}$ for $\mathrm{Ar}^{37}$ Cohesive energies and $\mathrm{MBC}$ are displayed in Fig. 3 as a function of interatomic distance for $C_{6}$-corrected and uncorrected DFT functionals. In order to obtain an equilibrium reference estimate of the MBC of $6.3 \mathrm{meV}$ ( $6.4 \%$ of the cohesive energy), we have used the two-body-crystal curve (dotted in Fig. 3) corresponding to $\operatorname{CCSD}(\mathrm{T}),{ }^{55}$ and the experimental equilibrium value ${ }^{61}$ [the zero-point energy (ZPE), estimated in Ref. 37, being removed]. The magnitude of the MBC DFT error becomes evident when inspecting the $C_{6}$ uncorrected cohesive energies in Table I: the $\mathrm{MBC}$ to the cohesive energies ranges from $\approx 35,50$, to $80 \%$ for $\mathrm{LDA}, \mathrm{PBE}$, and TPSS, respectively. PBE0, however, exhibits an absolute MBC of only $1.8 \mathrm{meV}$ due to the combination of the overestimated

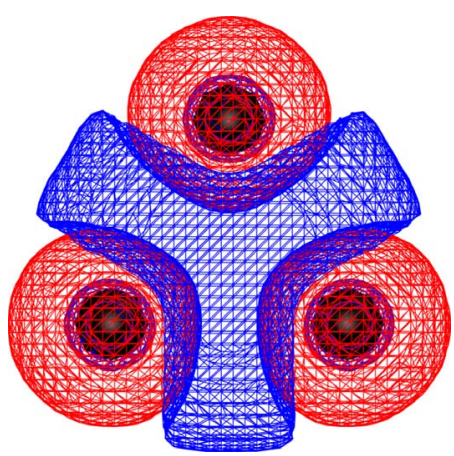

(a)

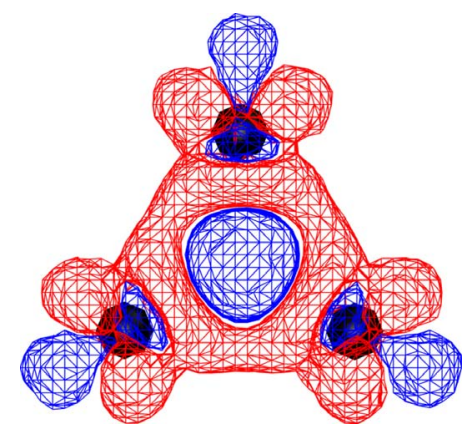

(b)

FIG. 2. (Color online) Electron density difference isosurface plots for $\mathrm{Ar}_{3}$ at $3 \AA$ equilateral distance-roughly the distance at which the three-body energy of $\mathrm{Ar}_{3}$ is maximal for LDA (see Fig. 1). Blue/dark gray represents depletion, red/gray represents creation of density. (a) Trimer density difference between CCSD, using the $Z$-vector method (Ref. 49) and LDA, $n_{\mathrm{CCSD}}-n_{\mathrm{LDA}}$, isovalue (1.0E-4 a.u.). (b) three-body LDA electron density, $n_{(3)}(\mathbf{r})$ $=n_{\text {trim }}(\mathbf{r})-3 n_{(2)}(\mathbf{r})-3 n_{\text {mon }}(\mathbf{r}) . n_{(2)}(\mathbf{r})$ being the two-body electron density, $n_{\text {dim }}(\mathbf{r})-2 n_{\text {mon }}(\mathbf{r})$. Isovalue (1.0E-5 a.u.). 


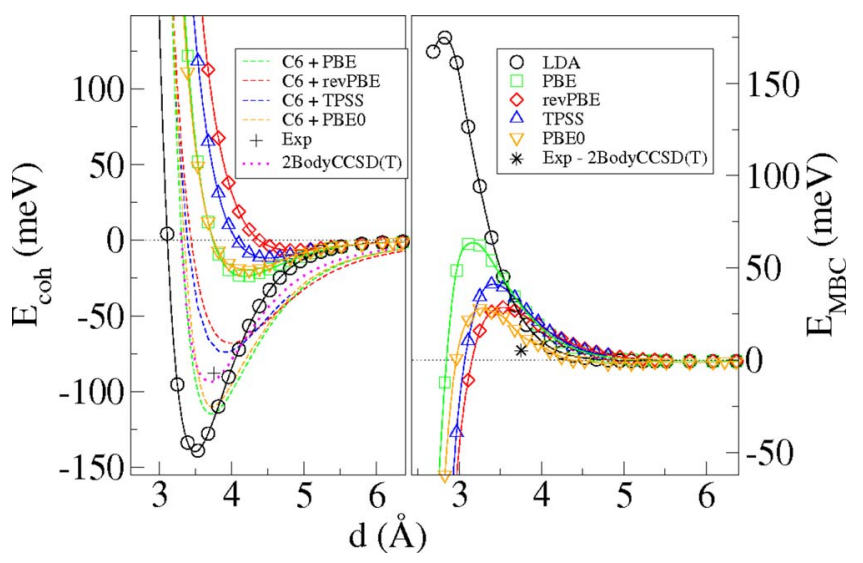

FIG. 3. (Color online) Left: Cohesive energies as a function of interatomic distance in the fcc crystal of Ar. The zero-point energy taken from Ref. 37 was removed from the experimental value (Ref. 61). DFT (solid) and $C_{6}+$ DFT (dashed) curves are displayed, together with the two-body-crystal curve (dotted) corresponding to $\operatorname{CCSD}(\mathrm{t})$ from Ref. 55. Right: Many-body curves corresponding to the difference between the cohesive energy (shown left) and the DFT two-body-crystal curves (not shown).

lattice constant with the fast decay of its MBC with the lattice constant.

Also, as in the case of the trimer, when using the $C_{6}$ correction for the GGA, meta-GGA, and hybrid functionals, binding properties, i.e., lattice constants and cohesive energies, improve consistently, while the MBC error worsens considerably. The two-body crystal is predicted best when using TPSS $+C_{6}$, the corresponding experimental cohesive energy, however, is still underestimated by $\approx 15 \%(14 \mathrm{meV})$, and the nearest-neighbor distance is $0.13 \AA$ longer than the experimental value of $3.78 \AA$. For all functionals, the deviation of the $\mathrm{MBC}$ from reference aggravates much more dramatically due to the $C_{6}$ than in the case of the trimer. For TPSS $+C_{6}$, it increases from 3.5 to $16.6 \mathrm{meV}$ due to the shortening of the Ar lattice constant resulting from the $C_{6}$ force field. If this $\mathrm{MBC}$ error was removed the TPSS $+C_{6}$ cohesive energy would be $-90.9 \mathrm{meV}$, very close to the experimental cohesive energy of $-88.0 \mathrm{meV}$ (ZPE being removed). Furthermore, one could expect that a correction of the repulsive $\mathrm{MBC}$ error would also support a decrease in the lattice constant toward the experimental value. Interestingly, while revPBE $+C_{6}$ is very close to $\mathrm{TPSS}+C_{6}, \mathrm{PBE} 0+C_{6}$ overestimates the cohesive energy, implying that the removal of the MBC error would only worsen the cohesive energy of $\mathrm{PBE} 0+C_{6}$. Therefore, and in spite of the absolute MBC error being smaller for PBE0 and revPBE than for TPSS, TPSS $+C_{6}$ appears to yield the most reasonable results.

\section{Ne and Kr}

To assess if the observed DFT trends for Ar are general, we have also investigated trimers of $\mathrm{Ne}$ and $\mathrm{Kr}$. The corresponding interatomic two- and three-body potentials are displayed in Fig. 4. Again, the dimer is inaccurately described by DFT, exhibiting similar trends as for Ar (Fig. 1). When inspecting the three-body DFT potentials of Ne and $\mathrm{Kr}$ (right

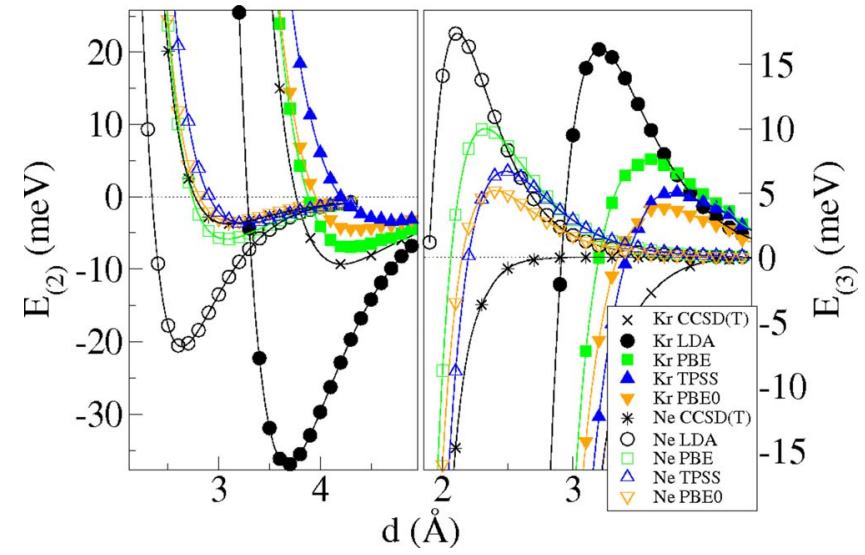

FIG. 4. (Color online) Left: Interaction energies as a function of interatomic distance in the $\mathrm{Ne}$ and $\mathrm{Kr}$ dimer. Right: three-body energy curves as a function of the interatomic distance in the equilateral $\mathrm{Ne}$ and $\mathrm{Kr}$ trimer.

hand plot in Fig. 4), it becomes clear that not only similarly erroneous trends as for Ar persist, but that their relative deviation from the reference is even more pronounced. Specifically, the $\operatorname{CCSD}(\mathrm{T})$ three-body curves exhibit extremely small repulsive maxima of less than $0.1 \mathrm{meV}$ at $\approx 3.3$ and $5.1 \AA$ for $\mathrm{Ne}$ and $\mathrm{Kr}$, respectively. It does not seem unreasonable to expect that, in complete analogy to Ar, DFT calculations of the bulk of $\mathrm{Ne}$ and $\mathrm{Kr}$ will also contain significant errors in the MBC.

\section{CONCLUSION}

We have found that across "Jacob's ladder" commonly applied parametrizations of KS-DFT result into strong and systematic overestimations of the repulsive three-body interactions in rare gases, prototypical van der Waals systems. For the bulk of Ar, the remaining deviation of the cohesive energy from experiment $(\approx 14 \mathrm{meV})$ compares best to the $\mathrm{MBC}$ error of TPSS $+C_{6}, \approx 17 \mathrm{meV}$. Such errors are at least six times larger than the accuracy required for predicting the energetic ranking of molecular crystal polymorphs, $2 \mathrm{meV}$, according to Refs. 62-64. The strong interatomic distance dependence of this error, as well as its variation between $\mathrm{Ne}$, $\mathrm{Ar}$, and $\mathrm{Kr}$, indicates that even for relative cohesive energies, one should not assume this error to cancel. It is therefore questionable if any intermolecular DFT + two-body approach for the parametrizations of interatomic or intermolecular forces is sufficient, and if, for example, the successful energy ranking of molecular crystals using $\mathrm{DFT}+C_{6}$ is rather fortuitous than to be expected. ${ }^{64}$

Furthermore, the "paradox" of the remarkable performance of LDA in describing solid state properties and in failing to describe molecular systems seems-at least partially - to be due to the fortuitous cancellation of $\mathrm{MBC}$ errors with two-body errors in the bulk. If the self-interaction error $^{65}$ is at the origin of this error and might be removed by corrections $^{66}$ remains an open question, in particular having Magyar et al. ${ }^{67}$ findings in mind that the electronic structure of rare gas solids does not improve upon using exact exchange functionals. 
Finally, we note that in contrast to using the DFT $+C_{6}$ correction for the underbonded dimer, a corresponding internuclear three-atom Axilrod-Teller potential would be unable to remedy this error since this term is repulsive for the equilateral triangle ${ }^{42}$ while an attractive correction would be required to reduce the overly repulsive DFT three-body curves-notwithstanding the finding that the Axilrod-Teller correction might be insufficient for the condensed phase anyway ${ }^{68}$ We hope that our findings will help to define further design criteria for the development of new density functionals. ${ }^{69}$

\section{ACKNOWLEDGMENTS}

Helpful comments and remarks by K. Leung, A. E. Mattsson, A. Oganov, M. Scheffler, P. A. Schultz, and A. Thompson are acknowledged. A.T. thank the Alexander von Humboldt $(\mathrm{AvH})$ foundation for financial support. O.A.vL. acknowledges support from SNL Truman Program LDRD Project No. 120209. Sandia is a multiprogram laboratory operated by Sandia Corporation, a Lockheed Martin Co., for the United States Department of Energy's National Nuclear Security Administration under Contract No. DE-AC0494AL85000. *tkatchen@fhi-berlin.mpg.de

†URL: http://www.sandia.gov/ oavonli, oavonli@sandia.gov

${ }^{1}$ P. Hohenberg and W. Kohn, Phys. Rev. 136, B864 (1964).

${ }^{2}$ W. Kohn and L. J. Sham, Phys. Rev. 140, A1133 (1965).

${ }^{3}$ D. M. Ceperley and B. J. Alder, Phys. Rev. Lett. 45, 566 (1980).

${ }^{4}$ J. P. Perdew, K. Burke, and M. Ernzerhof, Phys. Rev. Lett. 77, 3865 (1996).

${ }^{5}$ Y. Zhang and W. Yang, Phys. Rev. Lett. 80, 890 (1998).

${ }^{6}$ J. Tao, J. P. Perdew, V. N. Staroverov, and G. E. Scuseria, Phys. Rev. Lett. 91, 146401 (2003).

${ }^{7}$ J. P. Perdew, M. Ernzerhof, and K. Burke, J. Chem. Phys. 105, 9982 (1996).

${ }^{8}$ V. N. Staroverov, G. E. Scuseria, J. Tao, and J. P. Perdew, Phys. Rev. B 69, 075102 (2004).

${ }^{9}$ V. N. Staroverov, G. E. Scuseria, J. Tao, and J. P. Perdew, J. Chem. Phys. 119, 12129 (2003).

${ }^{10}$ S. Kristyán and P. Pulay, Chem. Phys. Lett. 229, 175 (1994).

${ }^{11}$ J. M. Pérez-Jordá and A. D. Becke, Chem. Phys. Lett. 233, 134 (1995).

${ }^{12}$ Y. Andersson, D. C. Langreth, and B. I. Lundqvist, Phys. Rev. Lett. 76, 102 (1996).

${ }^{13}$ M. Dion, H. Rydberg, E. Schröder, D. C. Langreth, and B. I. Lundqvist, Phys. Rev. Lett. 92, 246401 (2004).

${ }^{14}$ E. Engel, A. Höck, and R. M. Dreizler, Phys. Rev. A 61, 032502 (2000).

${ }^{15}$ S. Kümmel and L. Kronik, Rev. Mod. Phys. 80, 3 (2008).

${ }^{16}$ O. A. von Lilienfeld, I. Tavernelli, U. Rothlisberger, and D. Sebastiani, Phys. Rev. Lett. 93, 153004 (2004).

${ }^{17}$ O. A. von Lilienfeld-Toal, Ph.D thesis, EPFL (Switzerland), 2005.

${ }^{18}$ O. A. von Lilienfeld, I. Tavernelli, U. Rothlisberger, and D. Sebastiani, Phys. Rev. B 71, 195119 (2005).

${ }^{19}$ A. Tkatchenko and O. A. von Lilienfeld, Phys. Rev. B 73, 153406 (2006).

${ }^{20}$ I. Chun Lin, M. D. Coutinho-Neto, C. Felsenheimer, O. A. von Lilienfeld, I. Tavernelli, and U. Rothlisberger, Phys. Rev. B 75, 205131 (2007).

${ }^{21}$ Y. Zhao and D. G. Truhlar, J. Phys. Chem. A 109, 5656 (2005).

${ }^{22}$ W. Heitler and F. London, Z. Phys. 44, 455 (1927).

${ }^{23}$ R. Eisenschitz and F. London, Z. Phys. 60, 491 (1930).

${ }^{24}$ J. Hepburn, G. Scoles, and R. Penco, Chem. Phys. Lett. 36, 451 (1975).

${ }^{25}$ R. LeSar, J. Phys. Chem. 88, 4272 (1984).
${ }^{26}$ M. Elstner, P. Hobza, T. Frauenheim, S. Suhai, and E. Kaxiras, J. Chem. Phys. 114, 5150 (2001).

${ }^{27}$ X. Wu, M. C. Vargas, S. Nayak, V. Lotrich, and G. Scoles, J. Chem. Phys. 115, 8748 (2001).

${ }^{28} \mathrm{Q}$. Wu and W. Yang, J. Chem. Phys. 116, 515 (2002).

${ }^{29}$ D. Sanches-Portal, P. Ordejon, E. Artacho, and J. M. Soler, Int. J. Quantum Chem. 65, 453 (1997).

${ }^{30}$ M. Elstner, D. Porezag, G. Jungnickel, J. Elsner, M. Haugk, T. Frauenheim, S. Suhai, and G. Seifert, Phys. Rev. B 58, 7260 (1998).

${ }^{31}$ F. Ortmann, F. Bechstedt, and W. G. Schmidt, Phys. Rev. B 73, 205101 (2006).

${ }^{32}$ M. Hasegawa and K. Nishidate, Phys. Rev. B 70, 205431 (2004).

${ }^{33}$ S. Grimme, J. Comput. Chem. 25, 1463 (2004).

${ }^{34}$ U. Zimmerli, M. Parrinello, and P. Koumoutsakos, J. Chem. Phys. 120, 2693 (2004).

${ }^{35}$ A. J. Stone, The Theory of Intermolecular Forces (Oxford University Press, New York, 1996).

${ }^{36}$ I. G. Kaplan, Intermolecular Interactions: Physical Picture, Computational Methods and Model Potentials (Wiley, New York, 2006).

${ }^{37}$ K. Rościszewski, B. Paulus, P. Fulde, and H. Stoll, Phys. Rev. B 62, 5482 (2000).

${ }^{38}$ F. Ortmann, W. G. Schmidt, and F. Bechstedt, Phys. Rev. Lett. 95, 186101 (2005).

${ }^{39}$ M. A. Neumann and M.-A. Perrin, J. Phys. Chem. B 109, 15531 (2005).

${ }^{40}$ S. Grimme, J. Antony, T. Schwabe, and C. Mück-Lichtenfeld, Org. Biomol. Chem. 5, 741 (2007).

${ }^{41}$ S. Grimme, C. Mück-Lichtenfeld, and J. Antony, J. Phys. Chem. C 111, 11199 (2007).

${ }^{42}$ B. M. Axilrod and E. Teller, J. Chem. Phys. 11, 299 (1943).

${ }^{43}$ J. Hutter et al., computer code CPMD, Version 3.11, Copyright IBM Corp. 1990-2006, Copyright MPI-FKF Stuttgart 19972001 (http://www.cpmd.org).

${ }^{44}$ S. Goedecker, M. Teter, and J. Hutter, Phys. Rev. B 54, 1703 (1996).

${ }^{45}$ M. Krack, Theor. Chim. Acta 114, 145 (2005).

${ }^{46}$ E. J. Bylaska et al., NWChem A Computational Chemistry Package for Parallel Computers Version 5.0 (Pacific Northwest National Laboratory, Richland, Washington, 2006).

${ }^{47}$ T. H. Dunning, Jr., J. Chem. Phys. 90, 1007 (1989). 
${ }^{48}$ D. E. Woon and T. H. Dunning, Jr., J. Chem. Phys. 98, 1358 (1993).

${ }^{49}$ M. J. Frisch, G. W. Trucks, H. B. Schlegel, G. E. Scuseria, M. A. Robb, J. R. Cheeseman, J. A. Montgomery, Jr., T. Vreven, K. N. Kudin et al., computer code GAUSSIAN 03, Revision B.05, 2003.

${ }^{50}$ G. Kresse and J. Hafner, Phys. Rev. B 47, 558 (1993);http:// cms.mpi.univie.ac.at/vasp/

${ }^{51}$ S. Grimme, J. Comput. Chem. 27, 1787 (2006).

${ }^{52}$ P. Jurecka, J. Cerny, P. Hobza, and D. R. Salahub, J. Comput. Chem. 28, 555 (2007).

${ }^{53}$ A. Tkatchenko, V. Blum, and M. Scheffler (unpublished).

${ }^{54}$ P. Jurecka, J. Sponer, J. Cerny, and P. Hobza, Phys. Chem. Chem. Phys. 8, 1985 (2006).

${ }^{55}$ P. Slavicek, R. Kalus, P. Paska, I. Odvarkova, P. Hobza, and A. Malijevsky, J. Chem. Phys. 119, 2102 (2003).

${ }^{56}$ R. Podeszwa and K. Szalewicz, J. Chem. Phys. 126, 194101 (2007).

${ }^{57}$ J. Tao and J. P. Perdew, J. Chem. Phys. 122, 114102 (2005).

${ }^{58}$ P. R. Herman, P. E. LaRocque, and B. P. Stoicheff, J. Chem.
Phys. 89, 4535 (1988)

${ }^{59}$ J. F. Ogilvie and F. Y. H. Wang, J. Mol. Struct. 273, 277 (1992).

${ }^{60}$ O. G. Peterson, D. N. Batchelder, and R. O. Simmons, Phys. Rev. 150, 703 (1966).

${ }^{61}$ L. A. Schwalbe, R. K. Crawford, H. H. Chen, and R. A. Aziz, J. Chem. Phys. 66, 4493 (1977).

${ }^{62}$ J. D. Dunitz and H. A. Scheraga, Angew. Chem., Int. Ed. 44, 1766 (2005).

${ }^{63}$ P. G. Karamertzanis and S. L. Price, J. Chem. Theory Comput. 2, 1184 (2006).

${ }^{64}$ M. A. Neumann, F. J. J. Leusen, and J. Kendrick, Angew. Chem., Int. Ed. 47, 2427 (2008).

${ }^{65}$ J. P. Perdew and A. Zunger, Phys. Rev. B 23, 5048 (1981).

${ }^{66}$ M. M. Rieger and P. Vogl, Phys. Rev. B 52, 16567 (1995).

${ }^{67}$ R. J. Magyar, A. Fleszar, and E. K. U. Gross, Phys. Rev. B 69 , 045111 (2004).

${ }^{68}$ A. G. Donchev, J. Chem. Phys. 125, 074713 (2006).

${ }^{69}$ A. E. Mattsson, Science 122, 114102 (2002). 